\title{
Density functionals from many-body perturbation theory: The band gap for semiconductors and insulators
}

\author{
Myrta Grüning ${ }^{\text {a) }}$ \\ Donostia International Physics Center, E-20018 San Sebastián, Spain and European Theoretical \\ Spectroscopy Facility (ETSF), Universidad del País Vasco/Euskal Herriko Unibertsitatea, \\ E-20018 San Sebastián, Spain \\ Andrea Marini \\ Istituto Nazionale per la Fisica della Materia e Dipartimento di Fisica dell'Università di Roma \\ “Tor Vergata,"via della Ricerca Scientifica, I-00133 Roma, Italy and European Theoretical \\ Spectroscopy Facility (ETSF), Universidad del País Vasco/Euskal Herriko Unibertsitatea, \\ E-20018 San Sebastián, Spain \\ Angel Rubio ${ }^{\text {b) }}$ \\ Institut für Theoretische Physik Freie Universität Berlin, Arnimalle 14, D-14195 Berlin, Germany \\ and European Theoretical Spectroscopy Facility (ETSF), Universidad del País Vasco/Euskal Herriko \\ Unibertsitatea, E-20018 San Sebastián, Spain
}

(Received 31 January 2006; accepted 28 February 2006; published online 21 April 2006)

\begin{abstract}
Theoretically the Kohn-Sham band gap differs from the exact quasiparticle energy gap by the derivative discontinuity of the exchange-correlation functional. In practice for semiconductors and insulators the band gap calculated within any local or semilocal density approximations underestimates severely the experimental energy gap. On the other hand, calculations with an "exact" exchange potential derived from many-body perturbation theory via the optimized effective potential suggest that improving the exchange-correlation potential approximation can yield a reasonable agreement between the Kohn-Sham band gap and the experimental gap. The results in this work show that this is not the case. In fact, we add to the exact exchange the correlation that corresponds to the dynamical (random phase approximation) screening in the $G W$ approximation. This accurate exchange-correlation potential provides band structures similar to the local density approximation with the corresponding derivative discontinuity that contributes $30 \%-50 \%$ to the energy gap. Our self-consistent results confirm substantially the results for $\mathrm{Si}$ and other semiconductors obtained perturbatively [R. W. Godby et al., Phys. Rev. B 36, 6497 (1987)] and extend the conclusion to $\mathrm{LiF}$ and $\mathrm{Ar}$, a wide-gap insulator and a noble-gas solid. (C) 2006 American Institute of Physics. [DOI: 10.1063/1.2189226]
\end{abstract}

\section{INTRODUCTION}

Kohn-Sham (KS) density-functional ${ }^{1,2}$ ground-state calculations on a periodic system give as a by-product the oneelectron band structure. Because of the efficiency and the simplicity of the KS density functional method it is desirable to use the KS one-electron band structure for estimating excitation energies (quasiparticle dispersion) and in particular energy gaps in solids. However, the KS band gap $\varepsilon_{\text {gap }}^{\mathrm{KS}}$, given by the difference of the lowest unoccupied and the highest occupied KS orbital energies of an $N$-electron system

$$
\varepsilon_{\text {gap }}^{\mathrm{KS}}=\varepsilon_{N+1}(N)-\varepsilon_{N}(N)
$$

differs theoretically ${ }^{3-6}$ from the real energy gap

\footnotetext{
${ }^{a}$ Present address: Université Catholique de Louvain, Unité de PhysicoChimie et de Physique des Matériaux, B-1348 Louvain-la-Neuve, Belgium

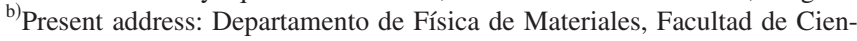
cias Químicas, UPV/EHU, Centro Mixto CSIC-UPV/EHU, and Donostia International Physics Center (DIPC), E-20018 San Sebastián, Spain
}

$$
E_{\text {gap }}=I-A
$$

that is obtained from the difference between the electron affinity $A$ and the first ionization potential $I$. While for systems with a finite number of electrons, e.g., atoms and molecules, the energy gap can be evaluated efficiently from the total energy difference,

$$
E_{\text {gap }}=E(N+1)+E(N-1)-2 E(N) .
$$

This technique cannot be directly applied to systems with an infinite number of electrons and one has to resort to other types of calculation to access this quantity.

The difference between $E_{\text {gap }}$ and $\varepsilon_{\text {gap }}^{\mathrm{KS}}$ is due ${ }^{7}$ to the derivative discontinuity $\Delta_{\mathrm{xc}}$ in the exchange correlational functional, ${ }^{3-6}$

$$
E_{\text {gap }}=\varepsilon_{\text {gap }}^{\mathrm{KS}}+\Delta_{\mathrm{xc}} .
$$

The question is then how large is the contribution $\Delta_{\mathrm{xc}}$ to the $E_{\text {gap }}$, or, in other words, whether or not it is still possible to estimate $E_{\text {gap }}$ from $\varepsilon_{\text {gap }}^{\mathrm{KS}}$ in real systems. The answer to this question is of paramount relevance for devising new approximations to the exchange-correlation potential and ker- 
nel in time-dependent density-functional theory. Indeed, there are well-known problems in calculations of response properties of extended systems directly related to the KS gap underestimation: the downward shift in energy of the optical absorption spectra of semiconductors and solids, the underestimation of long-range charge-transfer excitations ${ }^{8-10}$ and the overestimation of the (hyper)polarizabilities of polymeric chains. ${ }^{11,12}$ In fact the KS energy differences enter the timedependent calculations as the "zero-order approximation" for the excitation energies, while the "corrections" that shift the KS energy differences to the excitation energies should come from the exchange-correlation kernel. Therefore, determining the relative contribution of the derivative discontinuity $\Delta_{\mathrm{xc}}$ and the KS band gap $\varepsilon_{\text {gap }}^{\mathrm{KS}}$ to the energy gap $E_{\text {gap }}$ [Eq. (1.4)], and more in general to the excitation energies, would help to assess not only the approximation for the potential, but eventually also the approximation for the kernel. In particular, it would help to understand whether the downwards shift in the optical absorption spectra of semiconductors and insulators is due to deficiencies of the exchange-correlation potential or of the corresponding kernel.

For semiconductors and insulators the KS band gap calculated with the local density approximation (LDA) for the exchange-correlation potential are about 30\%-100\% lower than the experimental energy gap. ${ }^{13,14}$ Adding gradient corrections to the exchange-correlation potential does not improve the results for the band gaps. A longstanding discussion $^{15-18}$ is whether this large underestimation is an artefact of the LDA (and similar approximations) or is due to the $\Delta_{\mathrm{xc}}$.

A definitive answer can be given only when the exact $\mathrm{KS}$ potential is known for real systems. For atoms and small molecules the exact, or very accurate, KS exchangecorrelation potential has been obtained from the density calculated by quantum Monte Carlo or other accurate wavefunction methods (see, e.g., Refs. 19 and 20). However, for extended systems this technique has been applied only to a model semiconductor. ${ }^{21}$

An alternative way to obtain an accurate exchangecorrelation potential that can be feasible both for finite and for extended systems is the optimized effective potential (OEP) approach. ${ }^{22}$ Recent calculations for semiconductors employing the OEP method at the exchange-only level (the exact exchange potential) provided very good agreement between the KS and the interacting energy gap $E_{\text {gap }}{ }^{23,24}$ However, for noble-gas solids ${ }^{25}$ the exact exchange (EXX) potential, even though yielded larger KS band gap than the LDA potential, heavily underestimate the experimental gaps.

These results may suggest that a more sophisticated approximation for the exchange-correlation potential could provide a good estimate for the energy gap. In particular, an accurate correlation potential could further open the KS band gap for wide-gap insulators. On the other hand, the experience based on many-body perturbation theory (MBPT) suggests that Coulomb interaction tends to close the bare exchange band gap (see, e.g., Ref. 26).

In the present work we address this issue by deriving within the OEP approach the exchange-correlation potential corresponding to a given many-body energy functional (Sec. II A). In contrast to previous works ${ }^{23-25}$ we do not add a posteriori the correlation part of the LDA potential, but we treat the exchange and correlation at the same level of approximation. Sections III A and III B present the results for the band gaps and the derivative discontinuity of bulk $\mathrm{Si}$, $\mathrm{LiF}$, and Ar obtained with the potential corresponding to the $G W$ exchange-correlation self-energy. ${ }^{27,28}$ By comparison with the exchange-only Hartree-Fock (HF) results we show how the long-range correlation-included in the $G W$ selfenergy - affects the band gap and the derivative discontinuity. In fact, the long-range correlation reduces the exchangeonly band gap, and, in agreement with previous perturbative analyses, ${ }^{15,17}$ the band gaps we obtain are similar to the LDA band gaps.

\section{THEORETICAL FRAMEWORK}

\section{A. Density functionals from many-body perturbation theory}

Finding a reasonable approximation for the total energy as a functional of the electronic density is still a challenge in density-functional methods. The traditional way to derive density functionals is to use model systems, such as the homogeneous electron gas, where the energy is an explicit functional of the density. A different approach valid in general is to use energy expressions that are only implicit functional of the density, such as orbital-dependent functionals. For example, the orbital-dependent Hartree-Fock energy written in terms of $\mathrm{KS}$ orbitals is an implicit density functional, since the KS orbitals depends on the density (this procedure leads to the exact-exchange approach). Using an implicit density functional has the advantage that the physics of the electron-electron interaction is described via a nonlocal expression of the orbitals and the orbitals energy. With the OEP equation local potentials are derived from these nonlocal orbital/energy-dependent expressions.

In this work we follow the approach in Ref. 29 to derive the OEP equation from MBPT. Starting from $\Phi[G]$ - the universal functional of the Green's function $G$ that describes the nonclassical electron-electron interaction effects-different variational energy functionals are derived by adding functionals of the Green's function $G$ in a way that the total functional becomes the exact total energy of the system at the stationary point. This approach automatically implies the conservation laws for the number of particles, total energy, momentum, and angular momentum. ${ }^{30}$

The $\Phi[G]$ universal functional can be obtained from diagrammatic techniques of MBPT. ${ }^{31}$ Examples of $\Phi$ functional obtained in this way are the Hartree-Fock, the second-order Møller-Plesset (MP2), or the $G W$ approximations for the total energy. The functional derivative of $\Phi$ with respect to the Green's function gives the self-energy operator $\Sigma_{\mathrm{xc}}$

$$
\Sigma_{\mathrm{xc}}[G]=\frac{\delta \Phi[G]}{\delta G} .
$$




$$
G=G_{0}+G_{0} \Sigma_{\mathrm{xc}} G
$$

The self-energy operator $\Sigma_{\mathrm{xc}}$ contains the electron-electron interaction effects that leads the Hartree Green's function $G_{0}$ into the interacting Green's function $G$. In the Green's function framework, Eq. (2.2) should be solved self-consistently with similar equations for $\Sigma_{\mathrm{xc}}$ and for the screened interaction $W$ (Hedin's equations ${ }^{27}$ ). This approach is computationally unbearable and usually one approximates $\Sigma_{\mathrm{xc}}$ with the first order of Hedin's equations expanded in powers of the screened interaction $W$.

The variational nature of this formalism introduces some degree of arbitrariness in the derivation of the total energy functional. Indeed, there is not a unique way to construct variational energy functionals, and there exist different functional expressions, such as the Luttinger-Ward ${ }^{31}$ or the Klein $^{32}$ functionals. All these expressions satisfy Eq. (2.2) at the stationary point and give for this $G$ the same total energy. ${ }^{33}$

While this completes the general introduction to MBPTbased total energy functionals, in the present work we are interested in using these functionals within the KS density functional scheme, i.e., we are looking for local potentials from which the electronic properties are derived by solving the standard KS equations. ${ }^{34}$

A KS scheme from a variational MBPT energy functional is derived by restricting the variational freedom to $\mathrm{KS}$ one-electron Green's function $G_{s}$, that is, the noninteracting Green's function corresponding to the KS effective local potential $v_{s}$

$$
v_{s}(\mathbf{r})=v_{\mathrm{ext}}(\mathbf{r})+v_{H}(\mathbf{r})+v_{\mathrm{xc}}(\mathbf{r}),
$$

given by the sum of the external potential $v_{\text {ext }}$, of the classical Coulomb (Hartree) potential $v_{H}$, and of the exchangecorrelation potential $v_{\mathrm{xc}}$. Reference 29 showed that different choices for the variational energy functional lead to different OEP equations, in contrast to the full MBPT variational framework where the final Green's function is unique for all functionals. In this work we adopt the variational total energy functional proposed by Klein that leads to the "standard" and simplest form for the OEP equation and allows us to make a connection with previous works (in particular, it leads to the linearized Sham-Schlüter equation ${ }^{5}$ or equivalently, to the potential derived from the adiabatic connection formula using the RPA energy functional ${ }^{35}$ ).

Within the KS scheme the Klein functional looks like the standard expression for the KS total energy,

$E_{\mathrm{Klein}}^{\mathrm{KS}}=T_{s}+\frac{1}{2} \int d \mathbf{r} v_{H}(\mathbf{r}) n(\mathbf{r})+\int d \mathbf{r} v_{\mathrm{ext}}(\mathbf{r}) n(\mathbf{r})+i \Phi\left[G_{s}\right]$,

where $T_{s}$ is the kinetic energy of the KS independent particles, while the $\Phi$ is the exchange-correlation energy functional. One obtains the OEP integral equation by minimizing the KS-Klein density-functional energy expression with respect to the electron density,

$$
\begin{gathered}
\int d \omega \int d \mathbf{r}_{2} \int d \mathbf{r}_{3} G_{s}\left(\mathbf{r}, \mathbf{r}_{2}, \omega\right)\left[v_{\mathrm{xc}}\left(\mathbf{r}_{2}\right) \delta\left(\mathbf{r}_{2}-\mathbf{r}_{3}\right)\right. \\
\left.-\Sigma_{\mathrm{xc}}\left(\mathbf{r}_{2}, \mathbf{r}_{3} ; \omega\right)\right] G_{s}\left(\mathbf{r}_{3}, \mathbf{r}, \omega\right)=0 .
\end{gathered}
$$

The OEP integral equation [Eq. (2.5)] allows us to derive the local exchange-correlation potential $v_{\mathrm{xc}}$ corresponding to a $\Phi$-derived approximation of the self-energy operator $\Sigma_{\mathrm{xc}}$ [Eq. (2.1)].

Note that the $v_{\mathrm{xc}}$ derived in this way satisfies conservation laws for the particle number, the energy, the momentum, and the angular momentum (see Ref. 29 and references therein). Moreover the possibility of controlling the level of approximation of the potential by choosing the approximation for the $\Phi$ functional opens the way towards a systematic treatment of exchange-correlation effects in the KS densityfunctional scheme. For the exchange-only Hartree-Fock approximation Eq. (2.5) gives the OEP equation for the EXX potential, ${ }^{23,36}$ however, one can include correlation at-for example-the $G W$ or MP2 level by choosing the corresponding $\Phi$ functional in Eq. (2.4).

Besides $\Phi[G]$, another class of variational energy functionals can be constructed following Ref. 37 by starting from the universal functional $\Psi[G, W]$ of the Green's function and the screened interaction $W$. The advantage of the $\Psi[G, W]$ functional is that $W$ is treated as an independent variable, in particular, not dependent on $G$, and therefore it is possible to consider approximations that use simple model $W_{0}$ instead of solving the Dyson equation for $W$. Within this framework the simplest choice for the $\Psi[G, W]$ (Ref. 29) functional leads to the OEP equation in Eq. (2.5). However, different from the $\Phi$-derived OEP equation, the self-energy operator $\Sigma$ is a functional of both $G$ and $W$.

In this work we consider the local potentials corresponding to the Hartree-Fock (HF) and to the $G W_{0}$ approximations via the OEP equation [Eq. (2.5)]. Section II B presents the details of the solution of Eq. (2.5) in these two cases.

Finally, in contrast to local or semilocal density approximations for the energy functionals that give a zero derivative discontinuity, the exchange-correlation functionals derived from MBPT have a finite derivative discontinuity,

$$
\Delta_{\mathrm{xc}}(\mathbf{r})=\lim _{\delta \rightarrow 0}\left(\left.\frac{\delta E_{\mathrm{xc}}[n]}{\delta n(\mathbf{r})}\right|_{N+\delta}-\left.\frac{\delta E_{\mathrm{xc}}[n]}{\delta n(\mathbf{r})}\right|_{N-\delta}\right) .
$$

It has been shown ${ }^{38}$ that for solids the derivative discontinuity $\Delta_{\mathrm{xc}}$ of the potential corresponding to the $G W$ self-energy operator can be evaluated from the $N$-electron eigenvalues and eigenfunctions as

$$
\begin{aligned}
\Delta_{\mathrm{xc}}= & \left\langle\psi_{N+1}\left|\Sigma_{\mathrm{xc}}^{G W}\left(\varepsilon_{N+1}\right)-v_{\mathrm{xc}}\right| \psi_{N+1}\right\rangle \\
& -\left\langle\psi_{N}\left|\Sigma_{\mathrm{xc}}^{G W}\left(\varepsilon_{N}\right)-v_{\mathrm{xc}}\right| \psi_{N}\right\rangle .
\end{aligned}
$$

Note that this equation is similar to the standard perturbative solution of the Dyson equation in the $G W$ approximation besides the fact that it is evaluated at the KS eigenvalues instead of the quasiparticle energies. For a discussion of this point we refer the reader to Ref. 38. Thus, we can calculate within the same approach the KS band gap, the derivative discontinuity and, via Eq. (1.4), the energy gap. Similarly the complete quasiparticle levels can be inferred. The exchange- 
only case can be derived from Eq. (2.7) using $\Sigma_{\mathrm{xc}}=\Sigma_{x}^{\mathrm{HF}}$ and the $v_{\mathrm{xc}}=v_{x}{ }^{23,38}$ In what follows atomic units are used unless otherwise stated $(\hbar=m=e=1)$.

\section{B. Practical implementation of the OEP equation and computational details}

We discuss the implementation of the OEP method [Eq. (2.5)] for the HF self-energy $\Sigma_{x}^{\mathrm{HF}}$ and for the $G W$ approximation for the correlation part of $\Sigma_{\mathrm{xc}}^{G W}$, where

$\Sigma_{x}^{\mathrm{HF}}\left(\mathbf{r}, \mathbf{r}^{\prime}\right)=\frac{i}{2 \pi} \int d \omega^{\prime} e^{i \delta \omega^{\prime}} G_{s}\left(\mathbf{r}, \mathbf{r}^{\prime}, \omega^{\prime}\right) v\left(\left|\mathbf{r}-\mathbf{r}^{\prime}\right|\right)$

and

$\Sigma_{\mathrm{xc}}^{G W}\left(\mathbf{r}, \mathbf{r}^{\prime}, \omega\right)=\frac{i}{2 \pi} \int d \omega^{\prime} G_{s}\left(\mathbf{r}, \mathbf{r}^{\prime}, \omega+\omega^{\prime}\right) W\left(\mathbf{r}, \mathbf{r}^{\prime}, \omega^{\prime}\right)$.

Consistently with the discussion in Sec. II A, $\Sigma_{x}^{\mathrm{HF}}$ and $\Sigma_{\mathrm{xc}}^{G W}$ are evaluated at the $\mathrm{KS}$ Green functions $G_{s}$. We apply the OEP formalism to periodic systems only and consequently we expand the KS wave functions using a plane wave basis set $\mathbf{G}$ in the reciprocal space.

The "exact" exchange potential $v_{x}$ corresponding to $\Sigma_{x}^{\mathrm{HF}}$ [Eq. (2.8)] is obtained from the OEP equation [Eq. (2.5)] by substituting the representation of the KS Green's function $G_{S}$ in terms of the KS wave functions and eigenvalues

$$
G_{s}\left(\mathbf{r}, \mathbf{r}^{\prime}, \omega\right)=\sum_{n} \int_{\mathrm{BZ}} \frac{d \mathbf{k}}{(2 \pi)^{3}} \frac{\psi_{n \mathbf{k}}(\mathbf{r}) \psi_{n \mathbf{k}}^{*}\left(\mathbf{r}^{\prime}\right)}{\omega-\varepsilon_{\mathrm{nk}}+i \eta \operatorname{sgn}\left(\varepsilon_{\mathrm{nk}}-\mu\right)},
$$

where the integration runs over the first Brillouin zone (BZ) and the sum includes both occupied and empty states. By performing the integration on the frequencies in Eq. (2.5) we get

$$
\begin{aligned}
v_{x}(\mathbf{G})= & \sum_{\mathbf{G}^{\prime}} \widetilde{\chi}_{s}^{-1}\left(\mathbf{G}, \mathbf{G}^{\prime}\right) \\
& \times\left[4 \int_{\mathrm{BZ}} \frac{d \mathbf{k}}{(2 \pi)^{3}} \sum_{v c} \frac{\left\langle v \mathbf{k}\left|e^{i \mathbf{G}^{\prime} \cdot \mathbf{r}}\right| c \mathbf{k}\right\rangle\left\langle v \mathbf{k}\left|\Sigma_{x}\right| c \mathbf{k}\right\rangle}{\varepsilon_{c \mathbf{k}}-\varepsilon_{v \mathbf{k}}}\right],
\end{aligned}
$$

with $v$ and $c$ valence- and conduction-band indices. The matrix elements of the exchange self-energy operator are given by

$$
\begin{aligned}
\left\langle v \mathbf{k}\left|\Sigma_{x}\right| c \mathbf{k}\right\rangle= & -\sum_{v^{\prime}} \int_{\mathrm{BZ}} \frac{d \mathbf{q}}{(2 \pi)^{3}} \sum_{\mathbf{G}} 4 \pi \\
& \times \frac{\left\langle v \mathbf{k}\left|e^{i(\mathbf{q}+\mathbf{G}) \cdot \mathbf{r}}\right| v^{\prime} \mathbf{k}-\mathbf{q}\right\rangle\left\langle v^{\prime} \mathbf{k}-\mathbf{q}\left|e^{i(\mathbf{q}+\mathbf{G}) \cdot \mathbf{r}}\right| c \mathbf{k}\right\rangle}{|\mathbf{q}+\mathbf{G}|^{2}},
\end{aligned}
$$

with the integral in $\mathbf{q}$ discretized on a uniform grid and the $1 /|\mathbf{q}+\mathbf{G}|^{2}$ integrals evaluated on a small volume centered around each point in the discrete grid. ${ }^{39} \tilde{\chi}_{s}^{-1}\left(\mathbf{G}, \mathbf{G}^{\prime}\right)$ in Eq. (2.11) is the inverse of the reduced independent $\mathrm{KS}$ response function $\widetilde{\chi}_{s}\left(\mathbf{G}, \mathbf{G}^{\prime}\right)$,

$$
\begin{gathered}
\tilde{\chi}_{s}\left(\mathbf{G}, \mathbf{G}^{\prime}\right)=4 \int_{\mathrm{BZ}} \frac{d \mathbf{k}}{(2 \pi)^{3}} \sum_{v c} \frac{\left\langle v \mathbf{k}\left|e^{i \mathbf{G} \cdot \mathbf{r}}\right| c \mathbf{k}\right\rangle\left\langle c \mathbf{k}\left|e^{i \mathbf{G}^{\prime} \cdot \mathbf{r}}\right| v \mathbf{k}\right\rangle}{\varepsilon_{c \mathbf{k}}-\varepsilon_{v \mathbf{k}}}, \\
\text { for } \mathbf{G}, \mathbf{G}^{\prime} \neq 0
\end{gathered}
$$

$$
\tilde{\chi}_{s}\left(\mathbf{G}, \mathbf{G}^{\prime}\right)=0, \quad \text { for } \mathbf{G} \text { or } \mathbf{G}^{\prime}=0
$$

This redefinition of $\chi_{s}$ is necessary because the effective potential is defined up to a constant and consequently the KS response function is linear dependent. Therefore we have to project out the null space of the $\chi_{s}$ before undergoing the matrix inversion. A similar constraint is used in molecular calculations where the potential is fixed to be zero at infinite distance.

In the $G W$ approximation the dielectric function $\epsilon^{-1}$ reads

$$
\boldsymbol{\epsilon}^{-1}\left(\mathbf{r}, \mathbf{r}^{\prime}, \omega\right)=1+\int d \mathbf{r}_{1} v\left(\left|\mathbf{r}-\mathbf{r}_{1}\right|\right) \chi\left(\mathbf{r}_{1}, \mathbf{r}^{\prime}, \omega\right)
$$

that gives, when used in the expression for the screened interaction $W$,

$$
W\left(\mathbf{r}, \mathbf{r}^{\prime}, \omega\right)=\int d \mathbf{r}_{1} v\left(\left|\mathbf{r}-\mathbf{r}_{1}\right|\right) \epsilon^{-1}\left(\mathbf{r}^{\prime}, \mathbf{r}_{1}, \omega\right)
$$

The $\chi$ appearing in Eq. (2.14) is the full response function that we evaluate in the random phase approximation (RPA) [i.e., $\epsilon^{-1}=\left(1-v \chi_{s}\right)^{-1}$ ]. The frequency-dependent part of the $\epsilon^{-1}$ function is evaluated by considering a single plasmonpole model,

$$
\begin{aligned}
\epsilon_{\mathbf{G G}^{\prime}}^{-1}(\mathbf{q}, \omega) \approx & \delta_{\mathbf{G}^{\prime} \mathbf{G}^{\prime}}+\left[\frac{R_{\mathbf{G G}^{\prime}}(\mathbf{q})}{\omega-\widetilde{\omega}_{\mathbf{G G}^{\prime}}(\mathbf{q})+i \eta}\right. \\
& \left.-\frac{R_{\mathbf{G G}^{\prime}}(\mathbf{q})}{\omega+\widetilde{\omega}_{\mathbf{G G}^{\prime}}(\mathbf{q})-i \eta}\right],
\end{aligned}
$$

that is, it is assumed that there is only one excitation that takes all the oscillator strength at the frequency $\widetilde{\omega}$ with weight $R^{40}$

With these simplifications we can perform the integral in the frequency in Eq. (2.5) analytically for the correlation part of the exchange-correlation OEP potential $v_{c}$, 


$$
\begin{aligned}
v_{c}(\mathbf{G})= & \sum_{\mathbf{G}^{\prime}} \widetilde{\chi}_{s}^{-1}\left(\mathbf{G}, \mathbf{G}^{\prime}\right) \int_{\mathrm{BZ}} \frac{d \mathbf{k}}{(2 \pi)^{3}} \sum_{v c} \frac{\left\langle v \mathbf{k}\left|e^{i \mathbf{G}^{\prime} \cdot \mathbf{r}}\right| c \mathbf{k}\right\rangle\left\langle v \mathbf{k}\left|\Sigma_{e}\left(\varepsilon_{c \mathbf{k}}\right)+\Sigma_{h}\left(\varepsilon_{v \mathbf{k}}\right)\right| c \mathbf{k}\right\rangle}{\varepsilon_{c \mathbf{k}}-\varepsilon_{v \mathbf{k}}} \\
& +\sum_{v v^{\prime}} \frac{\left\langle v \mathbf{k}\left|e^{i \mathbf{G}^{\prime} \cdot \mathbf{r}}\right| v^{\prime} \mathbf{k}\right\rangle\left\langle v \mathbf{k}\left|\Sigma_{h}\left(\varepsilon_{v^{\prime} \mathbf{k}}\right)+\Sigma_{h}\left(\varepsilon_{v \mathbf{k}}\right)\right| v^{\prime} \mathbf{k}\right\rangle}{\varepsilon_{v^{\prime} \mathbf{k}}-\varepsilon_{v \mathbf{k}}}+\left.\sum_{v}\left\langle v \mathbf{k}\left|e^{i \mathbf{G}^{\prime} \cdot \mathbf{r}}\right| v \mathbf{k}\right\rangle \frac{\partial \Sigma_{h}}{\partial \omega}\right|_{\omega=\varepsilon_{v \mathbf{k}}} \\
& +\sum_{c c^{\prime}} \frac{\left\langle c \mathbf{k}\left|e^{i \mathbf{G}^{\prime} \cdot \mathbf{r}}\right| c \mathbf{k}\right\rangle\left\langle c \mathbf{k}\left|\Sigma_{e}\left(\varepsilon_{c \mathbf{k}}\right)+\Sigma_{e}\left(\varepsilon_{c^{\prime} \mathbf{k}}\right)\right| c^{\prime} \mathbf{k}\right\rangle}{\varepsilon_{c \mathbf{k}}-\varepsilon_{c^{\prime} \mathbf{k}}}+\left.\sum_{c}\left\langle c \mathbf{k}\left|e^{i \mathbf{G}^{\prime} \cdot \mathbf{r}}\right| c \mathbf{k}\right\rangle \frac{\partial \Sigma_{e}}{\partial \omega}\right|_{\omega=\varepsilon_{c \mathbf{k}}}
\end{aligned}
$$

As the correlation effects included in the standard $G W_{0}$ self-energy operator stems from the RPA for the screening we will refer to this potential as RPA. In Eq. (2.17) the $m n$ matrix elements of $\Sigma_{e}$ and of $\Sigma_{h}$ are

$$
\begin{aligned}
& \left\langle m \mathbf{k}\left|\Sigma_{e}\right| n \mathbf{k}\right\rangle=-\sum_{v^{\prime}} \int_{\mathrm{BZ}} \frac{d \mathbf{q}}{(2 \pi)^{3}} \sum_{\mathbf{G G}^{\prime}} 4 \pi \frac{R_{\mathbf{G G}^{\prime}}(\mathbf{q})}{\omega+\widetilde{\omega}_{\mathbf{G G}^{\prime}}(\mathbf{q})-i \eta} \times \frac{\left\langle m \mathbf{k}\left|e^{i(\mathbf{q}+\mathbf{G}) \cdot \mathbf{r}}\right| v^{\prime} \mathbf{k}-\mathbf{q}\right\rangle\left\langle n \mathbf{k}\left|e^{i(\mathbf{q}+\mathbf{G}) \cdot \mathbf{r}}\right| v^{\prime} \mathbf{k}-\mathbf{q}\right\rangle}{|\mathbf{q}+\mathbf{G}|^{2}}, \\
& \left\langle m \mathbf{k}\left|\Sigma_{h}\right| n \mathbf{k}\right\rangle=-\sum_{c^{\prime}} \int_{\mathrm{BZ}} \frac{d \mathbf{q}}{(2 \pi)^{3}} \sum_{\mathbf{G}} 4 \pi \frac{R_{\mathbf{G G}^{\prime}}(\mathbf{q})}{\omega-\widetilde{\omega}_{\mathbf{G G}^{\prime}}(\mathbf{q})+i \eta} \times \frac{\left\langle m \mathbf{k}\left|e^{i(\mathbf{q}+\mathbf{G}) \cdot \mathbf{r}}\right| c^{\prime} \mathbf{k}-\mathbf{q}\right\rangle\left\langle n \mathbf{k}\left|e^{i(\mathbf{q}+\mathbf{G}) \cdot \mathbf{r}}\right| c^{\prime} \mathbf{k}-\mathbf{q}\right\rangle}{|\mathbf{q}+\mathbf{G}|^{2}} .
\end{aligned}
$$

Note that the solution of Eq. (2.17) is computationally very expensive due to the double summation on conduction bands. Indeed the computational cost can be noticeably reduced by using a common-energy denominator type of approximation. ${ }^{41-44}$ We perform here the full solution of the OEP equation in order to clarify the impact of the self-energy corrections on the KS-OEP band gap.

In our implementation, the $\epsilon^{-1}$ function [Eq. (2.14)] and therefore $W$ [Eq. (2.15)] are not updated during the selfconsistent loop, but evaluated once from LDA wave functions and band energies. This approximation is justified within the $\Psi$ functional framework mentioned in Sec. II A. In Sec. III A we test the impact of this approximation by using different wave functions and band energies for the screening.

As the practical implementation concerns, the ABINIT (Ref. 45) code has been used to perform LDA calculations for bulk $\mathrm{Si}, \mathrm{LiF}$ and $\mathrm{Ar}$ (face-centered-cubic lattice at experimental lattice constant). Troullier-Martins ${ }^{46,47}$ norm conserving LDA pseudopotentials have been used and for $\mathrm{Si}, \mathrm{LiF}$, and Ar the plane wave cutoff in the LDA (Refs. 48 and 49) calculations is respectively 18,80 , and $70 \mathrm{Ry}$. For all systems the Brillouin zone is sampled with a $4 \times 4 \times 4$ Monkhorst-Pack ${ }^{50}$ grid.

The OEP-KS potentials have been implemented in the pseudopotential plane-wave code SELF. ${ }^{51}$ In the solution of the KS equations we used the LDA wave functions as basis set. In the EXX calculations we tested also the EXX selfconsistent norm conserving pseudopotentials ${ }^{52}$ The description of the core-valence interaction turns out to be not very important for the band structures of the systems considered in this work.

The solution of the OEP equations must be converged with the number of unoccupied states and $\mathbf{G}$ vectors in the summations appearing in Eqs. (2.11), (2.17), (2.13), and (2.12). In the case of the $v_{c}$ potential [Eq. (2.17)] the number the $\mathbf{G}$ vectors in the summation of the $\epsilon^{-1}$ function must be converged as well. For Si we used 100 unoccupied bands, $169 \mathbf{G}$ vectors in the OEP equation and $59 \mathbf{G}$ vectors for the $\epsilon^{-1}$ function. For LiF and Ar we used 200 unoccupied bands $331 \mathbf{G}$ vectors in the OEP equation, and $113 \mathbf{G}$ vectors in the $\epsilon^{-1}$ function. All the parameters entering the calculations have been carefully converged to yield a $0.1 \mathrm{eV}$ mean numerical uncertainty in the final band gaps.

\section{RESULTS AND DISCUSSION}

Previous calculations for semiconductors using the EXX [Eq. (2.11)] plus LDA correlation potential (EXX+LDA) suggest that this approximation can be used to predict the energy gap. ${ }^{24}$ In this section we discuss the effect on the KS band structure of adding either the LDA or the RPA for the correlation [Eq. (2.17)] to the EXX potential. Then we investigate the contribution of the derivative discontinuity to the energy gap in the EXX and the EXX+RPA approximations. For the prototype semiconductor $\mathrm{Si}$, and two wide-gap insulators, $\mathrm{LiF}$ and $\mathrm{Ar}$, we show that the EXX band gap is reduced by the addition of the RPA correlation and that the contribution of the derivative discontinuity cannot be neglected.

\section{A. The KS band gaps with EXX + LDA and EXX +RPA}

Table I compares the effect on the KS band gaps for $\mathrm{Si}$, $\mathrm{LiF}$, and Ar using either the RPA or the LDA correlation for the potential. The KS band gaps are calculated at high symmetry points in the Brilliouin zone, and compared with the experimental, the $G W$ and the LDA gap. In Fig. 1 we compare the full KS band structures calculated with EXX +LDA, EXX+RPA, and LDA. All the results presented in Table I and Fig. 1 are consistently obtained within the same 
TABLE I. Calculated Kohn-Sham (KS) band gaps for bulk Si, LiF, and Ar using the following exchangecorrelation potentials as discussed in the main text: EXX, EXX+LDA, EXX+RPA, and LDA (see Sec. III for abbreviation). The results are compared with the calculated energy gap in the $G W$ method and with experiments.

\begin{tabular}{|c|c|c|c|c|c|c|}
\hline & EXX & $\mathrm{EXX}+\mathrm{LDA}$ & $\mathrm{EXX}+\mathrm{RPA}$ & LDA & $G W$ & Exp. \\
\hline \multicolumn{7}{|l|}{$\mathrm{Si}$} \\
\hline$\Gamma \rightarrow \Gamma$ & 3.2 & 3.3 & 2.6 & 2.6 & 3.2 & 3.37 \\
\hline$\Gamma \rightarrow X$ & 1.5 & 1.6 & 0.6 & 0.7 & 1.2 & 1.25 \\
\hline$\Gamma \rightarrow L$ & 2.2 & 2.3 & 1.5 & 1.5 & 2.1 & $2.1,{ }^{\mathrm{a}} 2.4^{\mathrm{b}}$ \\
\hline \multicolumn{7}{|l|}{$\mathrm{LiF}$} \\
\hline$\Gamma \rightarrow \Gamma$ & 11.2 & 11.5 & 9.3 & 8.9 & 13.5 & $14.2^{\mathrm{c}}$ \\
\hline$\Gamma \rightarrow X$ & 16.9 & 17.2 & 15.3 & 14.8 & 20.1 & \\
\hline$\Gamma \rightarrow L$ & 13.0 & 13.3 & 11.1 & 10.6 & 15.3 & \\
\hline \multicolumn{7}{|l|}{$\mathrm{Ar}$} \\
\hline$\Gamma \rightarrow \Gamma$ & 9.7 & 10.1 & 8.8 & 8.2 & 13.1 & $14.19^{\mathrm{d}}$ \\
\hline$\Gamma \rightarrow X$ & 12.1 & 12.5 & 11.4 & 10.6 & 16.0 & \\
\hline$\Gamma \rightarrow L$ & 12.2 & 12.6 & 11.5 & 11.0 & 16.3 & \\
\hline
\end{tabular}

methodological framework described in previous section. This prevents spurious numerical effects due to different possible implementations.

For Si the EXX band gaps are in quite good agreement with the $G W$ results and the experimental data. ${ }^{53,54}$ The LDA correlation opens rigidly the EXX band gaps (EXX+LDA column) by only $0.1 \mathrm{eV}$ and therefore the results are still in satisfactory agreement with $G W$ and experimental energy gaps as it was found already in Ref. 23. Conversely, adding RPA correlation to EXX (EXX+RPA) closes strikingly the band gap and also modifies the overall band structure. With the EXX+RPA potential the band gap is smaller by 0.6-0.9 eV than the EXX band gaps and the results are very close to those obtained within the LDA. This result confirms substantially the results for Si found in Ref. 15 obtained with the EXX+RPA potential by the perturbative solution of the KS equations using the unperturbed LDA wave functions and eigenenergies. In fact, we found that the LDA wave functions are very close to the EXX+RPA wave functions and this justifies the success of the perturbation approach used in Ref. 15.

For LiF the EXX band gaps are about $2 \mathrm{eV}$ larger than the LDA, still underestimating by more than $2 \mathrm{eV}$ the $G W$
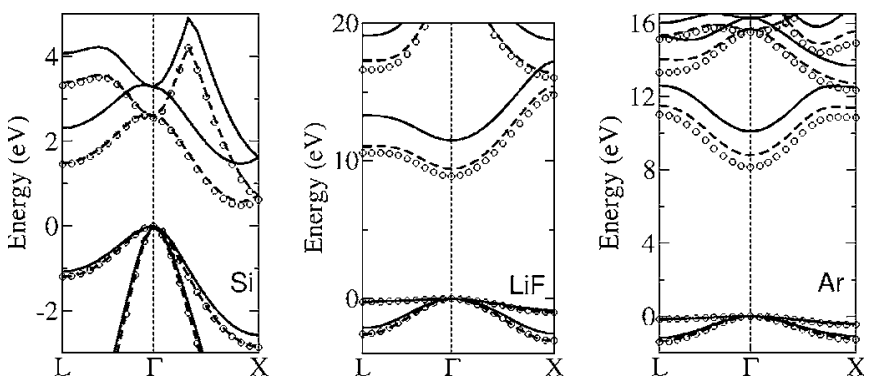

FIG. 1. Band structure of bulk $\mathrm{Si}, \mathrm{LiF}$, and $\mathrm{Ar}$ calculated with the EXX + LDA (continuous line), EXX+RPA (dashed line), and LDA (circles) approximations. The top of the valence band is set to $0 \mathrm{eV}$ for all the systems. and experimental ${ }^{55}$ values. The LDA correlation opens rigidly the EXX band gap by $0.3 \mathrm{eV}$. The effect of LDA correlation is small and therefore the EXX+LDA still largely underestimates the energy gap. As for $\mathrm{Si}$, adding RPA correlation to EXX closes the band gap and brings the results close to the LDA results. The band gaps found with EXX + RPA and LDA differs in fact by approximately $0.3 \mathrm{eV}$.

Bulk Ar shows a trend similar to LiF. The EXX potential improves the agreement with the experimental gap ${ }^{56}$ with respect to LDA, but the band gap is still underestimated by more than $4 \mathrm{eV}$. The LDA correlation in the EXX+LDA slightly opens the band gaps $(0.4 \mathrm{eV})$, while the RPA correlation closes the gap by $0.7-0.9 \mathrm{eV}$.

As discussed in Sec. II B, the screened Coulomb interaction $W$ [entering Eqs. (2.14) and (2.15)] is calculated with LDA eigenvalues and eigenfunctions and is not updated during the self-consistent loop. To estimate the effect of this approximation we have repeated the calculations using a screened interaction calculated with EXX eigenfunctions and eigenvalues. In fact, compared with LDA, EXX represents qualitatively the other extreme in the range of approximated KS eigenfunctions and eigenvalues. Table II shows that the screening calculated from EXX wave function is weaker and gives larger band gaps, but the effect is only of $0.1-0.2 \mathrm{eV}$. Since $W$ calculated from the EXX eigenfunctions and eigenvalues yields a band structure close to LDA as well, we conclude that $W$ can be safely approximated at the non-selfconsistent level with little effect on the final result.

To summarize, in all the systems we have studied, EXX gives a larger band gap than LDA, even if only for $\mathrm{Si}$, the $\operatorname{EXX}(+$ LDA $)$ band gap agrees with the experiment. As already observed in Ref. 25 the $\mathrm{EXX}(+\mathrm{LDA})$ scheme works well for $s p$ semiconductors, ${ }^{24}$ but heavily underestimates energy gaps in wide-gap insulators. The LDA correlation induces only a small rigid opening of the band gap: in Ref. 24 this result yielded to the conclusion that correlation was un- 
TABLE II. Comparison between the KS band gaps for bulk Si, LiF, and $\mathrm{Ar}$ calculated with the EXX+RPA exchange correlation potential using for the screened Coulomb interaction $W_{0}$ either the LDA or the EXX eigenfunctions and eigenvalues.

\begin{tabular}{|c|c|c|}
\hline & $\mathrm{EXX}+\mathrm{RPA}(\mathrm{LDA})$ & $\mathrm{EXX}+\mathrm{RPA}(\mathrm{EXX})$ \\
\hline \multicolumn{3}{|l|}{$\mathrm{Si}$} \\
\hline$\Gamma \rightarrow \Gamma$ & 2.6 & 2.6 \\
\hline$\Gamma \rightarrow X$ & 0.6 & 0.8 \\
\hline$\Gamma \rightarrow L$ & 1.5 & 1.5 \\
\hline \multicolumn{3}{|l|}{$\mathrm{LiF}$} \\
\hline$\Gamma \rightarrow \Gamma$ & 9.3 & 9.5 \\
\hline$\Gamma \rightarrow X$ & 15.3 & 15.6 \\
\hline$\Gamma \rightarrow L$ & 11.1 & 11.3 \\
\hline \multicolumn{3}{|l|}{$\mathrm{Ar}$} \\
\hline$\Gamma \rightarrow \Gamma$ & 8.8 & 8.9 \\
\hline$\Gamma \rightarrow X$ & 11.4 & 11.5 \\
\hline$\Gamma \rightarrow L$ & 11.5 & 11.6 \\
\hline
\end{tabular}

important for band gap with respect to the exchange. However, as emphasized in Refs. 57 and 58, the partitioning of exchange and correlation in the approximated (semi)local density functionals does not correspond to the partitioning in MBPT. The LDA for exchange contains long-range correlation effects that are missing in the LDA for correlation instead. As a consequence combining EXX, the "pure" exchange term, with the LDA correlation leads to an unbalanced approximation that misses long-range correlation effects.

The RPA correlation, on the other hand, embodies the long-range correlation effects (see, e.g., Ref. 59 and references therein) as confirmed by the results in Table I showing that RPA correlation has a crucial effect on the band structure. It corrects the exaggerated tendency of EXX potential to flatten the bands and it strikingly closes the band gap. The latter effect due to long-range correlation is already known: in the $G W$ method the long-range correlation induces the screening of the bare exchange and shrinks the gap. Similar results within the KS approach have been obtained in Ref. 60 with the potential corresponding to the screened exchangeCoulomb hole approximation for the self-energy, and in Ref. 15 with the EXX+RPA, but solving the KS equation perturbatively.

Finally, we stress that like in the exchange-only case, the EXX term in EXX+RPA energy cancels the self-interaction contribution due to the Hartree energy. The RPA correlation, even though introduces a minor error because of the selfpolarization effects in the screening, does not spoil this property. In spite of that, the EXX+RPA provides results for the band structure very close to the LDA whose exchange part is heavily affected by the self-interaction error. In fact the selfinteraction error in the LDA has been pointed out as the responsible for the underestimation of the band gaps (e.g., Refs. 23 and 24). We argue that the LDA results are close to the EXX+RPA since the self-interaction error in the LDA exchange accounts in part for long-range effects ${ }^{57}$ that are not counted, as mentioned above, in the LDA correlation. The resulting LDA potential is therefore similar to the sum

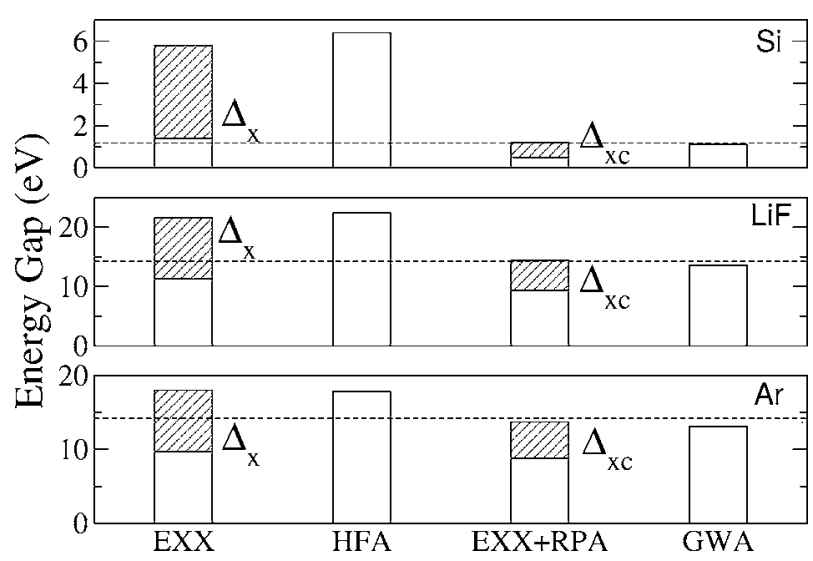

FIG. 2. Minimum-energy gap for bulk $\mathrm{Si}, \mathrm{LiF}$, and $\mathrm{Ar}$ as calculated with the EXX and EXX+RPA approximations within the KS density-functional framework (the white bar is the band-gap contribution, the striped bar is the derivative discontinuity contribution), and with the HF and $G W$ methods. The dashed line indicates the experimental value.

of the EXX and the RPA correlation. On the other hand $\operatorname{EXX}(+\mathrm{LDA})$ is self-interaction free (in exchange), but does not contain the long-range correlation effects and induces larger band gaps than the EXX+RPA.

The RPA potential constitutes just the first step in the MBPT series to include consistently correlation in the functionals and it is not the definitive answer to the problem. For example, it is still unclear how important it is to have a fully self-interaction free correlation potential.

\section{B. The derivative discontinuity and energy gap with EXX and EXX+RPA}

In the previous subsection we compared the KS band gap $\varepsilon_{\text {gap }}^{\text {KS }}$ with the $G W$ and experimental energy gap $E_{\text {gap }}$. However, as mentioned previously, the energy gap $E_{\text {gap }}^{\mathrm{KS}}$ within KS density-functional theory includes a contribution from the $E_{\mathrm{xc}}$ derivative discontinuity $\Delta_{\mathrm{xc}}$ [Eq. (1.4)]. In contrast to the (semi)local density approximations for the energy functionals, the EXX and EXX+RPA have a finite derivative discontinuity that can be calculated from Eq. (2.7). Figure 2 compares the energy gap, for EXX, EXX+RPA [Eq. (1.4)], and for the $\mathrm{HF}$ and $G W$ approximations.

For $\mathrm{Si}$, the EXX derivative discontinuity $\Delta_{x}$ is $4.5 \mathrm{eV}$, almost three times the EXX band gap $\varepsilon_{\text {gap }}^{\text {EXX }}$. The latter, as discussed in Sec. III A, is a good estimate of the experimental energy gap for Si. However, the addition of $\Delta_{x}$ to $\varepsilon_{\text {gap }}^{\mathrm{EXX}}$ gives an energy gap $E_{\text {gap }}^{\text {EXX }}$ of $\sim 6 \mathrm{eV}$, very close to the HF value, that severely overestimates the experimental value of $1.17 \mathrm{eV}$. On the other hand, the derivative discontinuity $\Delta_{\mathrm{xc}}$ corresponding to the EXX+RPA functional is much smaller than $\Delta_{x}$, in fact the RPA correlation reduces the discontinuity from 4.5 to $0.7 \mathrm{eV}$. The discontinuity $\Delta_{x c}$ summed to the $\mathrm{EXX}+\mathrm{RPA}$ band gap $\varepsilon_{\text {gap }}^{\mathrm{EXX}+\mathrm{RPA}}$ gives an energy gap of $1.2 \mathrm{eV}$ in very good agreement with the experimental value. For LiF the EXX derivative discontinuity $\Delta_{x}$ is $9 \mathrm{eV}$, almost $80 \%$ of the $\varepsilon_{\text {gap }}^{\mathrm{EXX}}$, and as a consequence $E_{\text {gap }}^{\mathrm{EXX}}$ overestimates the experimental energy gap by $6 \mathrm{eV}$. The derivative discontinuity $\Delta_{\mathrm{xc}}^{\mathrm{EXX}+\mathrm{RPA}}$ corresponding to EXX+RPA is instead about $5 \mathrm{eV}$ and $E_{\text {gap }}^{\mathrm{EXX}+\mathrm{RPA}}$ is $14.4 \mathrm{eV}$, a good estimate for the experimental value of $14.2 \mathrm{eV}$. Similarly, for Ar the EXX 
derivative discontinuity $\Delta_{x}$ is about $8 \mathrm{eV}$ and its addition to $\varepsilon_{\text {gap }}^{\text {EXX }}$ leads to an energy gap $E_{\text {gap }}^{\text {EXX }} 18 \mathrm{eV}$ that overestimates the experimental energy gap by $4 \mathrm{eV}$. The correlation reduces the exchange-only derivative discontinuity by $40 \%$ : $\Delta_{\mathrm{xc}}^{\mathrm{EXX}+\mathrm{RPA}}$ is about $5 \mathrm{eV}$. The $E_{\mathrm{gap}}^{\mathrm{EXX}+\mathrm{RPA}}$ is then $13.7 \mathrm{eV}$ and slightly underestimates the experimental value of $14.2 \mathrm{eV}$.

For all the systems the results for the energy gap within the EXX-KS and HF approach are close to each other. In fact $E_{\text {gap }}^{\mathrm{HF}}$ is obtained from the self-consistent solution of the HF equation while the $E_{\text {gap }}^{\mathrm{EXX}}$ is calculated by adding to the $\mathrm{KS}$ band gap the derivative discontinuity: the latter can be interpreted as the first-order perturbation correction to the EXX energies due to the perturbation that leads the KS into the HF equation. Note that the addition of LDA would not help to reduce the EXX energy gap. In fact, the LDA correlation increases slightly the band gap (see Table I) and it could contribute to the discontinuity only through the changes in the wave functions since there is no derivative discontinuity in the energy functional within the LDA. The total effect would be therefore negligible compared with the error on the energy gap.

As in the $G W$ method, in the EXX+RPA the dynamical screening of the Coulomb interaction reduces the energy gap with respect to the bare interaction case and provides a good estimate for the experimental band gap. The closeness of the $\mathrm{EXX}+\mathrm{RPA}$ and $G W$ results for the energy gap is not surprising: the $G W$ energy gap is obtained by adding to the LDA band gap the quasiparticle corrections that are calculated with an expression analogous to Eq. (2.7) with the difference that the self-energy operator is evaluated not at the KS, but at the quasiparticle energies. In fact, as shown in the previous subsection, the LDA and EXX+RPA band gap are very similar and the same holds for the wave functions of the highest occupied and the lowest conduction band. The difference between the $G W$ and EXX+RPA energy gap stems mostly from the energy dependence of the self-energy operator.

Considering EXX+RPA as an accurate approximation for the KS exchange-correlation potential we can conclude that, at least for the systems considered in this work, the $E_{\mathrm{xc}}$ derivative discontinuity contributes by $30 \%-50 \%$ to the energy gap and cannot be neglected when comparing the $\mathrm{KS}$ band gap with the real energy gap of a system. Note that for $\mathrm{Si}$, the difference between the EXX+RPA and EXX band gaps $\left(\varepsilon_{\text {gap }}^{\mathrm{EXX}+\mathrm{RPA}}-\varepsilon_{\text {gap }}^{\mathrm{EXX}}\right)$ is, with opposite sign, approximately equal to the derivative discontinuity $\Delta_{\mathrm{xc}}^{\mathrm{EXX}+\mathrm{RPA}}$ and as a result $\varepsilon_{\text {gap }}^{\text {EXX }} \sim E_{\text {gap }}$. Nevertheless this cancellation is fortuitous (even though it seems to hold in general for $s p$ semiconductors) as demonstrated by the results for $\mathrm{LiF}$, for Ar, and other noblegas solids. ${ }^{25}$

\section{CONCLUSIONS}

In this work we have addressed the bandgap problem in the standard KS scheme for $s p$ semiconductors $(\mathrm{Si})$, widegap insulators (LiF), and noble-gas solids (Ar). The KS band gap differs from the energy gap by the derivative discontinuity of the exchange-correlation functional. For real systems it is still not assessed how large this quantity is. In particular, recent calculations for semiconductors with EXX+LDA re- newed the hope that the derivative discontinuity could be small and that the KS density-functional method could be used in band-structure calculations.

Conversely, the results in this paper obtained with the accurate EXX+RPA potential, show that the derivative discontinuity contributes by as much as $30 \%-50 \%$ to the energy gap. Therefore the large difference between the LDA band gap and the experimental or quasiparticle energy gap is not due to an artefact of the approximation, but is mostly due to the derivative discontinuity. On the other hand, we have found that the good agreement of the EXX+LDA for $\mathrm{Si}$ is "fortuitous" due to neglecting both the correlation in the KS potential and contribution of the derivative discontinuity.

Both the EXX+RPA and EXX have been derived from the energy expression of the Klein functional of MBPT via the OEP method. The EXX+RPA corresponds to the $G W_{0}$ approximation for the self-energy, with the $W_{0}$ calculated within the RPA. In contrast to the EXX+LDA where the exchange is treated at OEP-MBPT level while the correlation is taken from the homogeneous electron gas model, in the EXX + RPA both exchange and correlation are treated at the same level of approximation. In fact the energy gap of $\mathrm{EXX}+\mathrm{LDA}$ - calculated by adding the corresponding derivative discontinuity to the band gap-is hugely overestimated, highlighting the imbalance of the approximation for exchange (large derivative discontinuity) and for the correlation (no derivative discontinuity). The energy gap calculated for EXX+RPA, on the other hand, is in quite good agreement with the experimental data once the exchangecorrelation derivative discontinuity is summed to the KS band gap.

The accuracy of the EXX+RPA potential could be improved by choosing a better approximation than $G W$ for the $\Phi$ functional, or by using a better approximation than RPA for the polarization, or by starting from a different energy functional (the one of Luttinger-Ward expression, for example, that leads to a different OEP equation ${ }^{61}$ ). However, the fact that EXX+RPA energy gap (band gap plus derivative discontinuity) provides a good estimate for the experimental gap makes us confident of the quality of this approximation. Moreover, even for improved approximations it remains valid that adding long-range correlation closes the EXX band gap. Since the EXX band gap already underestimates the energy gap for wide-gap insulators, we infer that it is not possible to find an approximation for the exchangecorrelation potential that successfully estimates the energy gap of wide-gap insulators and semiconductors.

For time-dependent calculations of optical absorption spectra, and in general of extended systems, the results we found indicate that the KS energy differences differ substantially from excitation energies. Therefore the correction that brings the KS energy differences to the excitation energies comes from the kernel, in particular, from the exchangecorrelation part since, unlike in finite systems, the Hartree part of the kernel does not correct the KS energy differences. Theoretical analyses ${ }^{62-66}$ on the properties of the kernel suggest that the correction to the KS orbital energies differences should come from a singularity in the frequency dependence of the exchange-correlation kernel. 
The theory employed in this work to derive accurate exchange-correlation potentials, can be applied as well for deriving accurate exchange-correlation kernels ${ }^{29,67}$ and could be a valuable tool to tackle this problem. Work in this direction is in progress.

\section{ACKNOWLEDGMENTS}

Work supported by the Nanoquanta Network of Excellence (NMP4-CT-2004-500198), Spanish MCyT and the Barcelona Supercomputer Center. One of the authors (A.R.) thanks the Humboldt Foundation under the 2005 Bessel research award. We benefited a lot from discussion and collaboration with Professor U. von Barth. We thank Dr. E. Engel for providing us with the code to generate the exact exchange pseudopotentials. We acknowledge useful discussion with Professor E.K.U. Gross, Dr. L. Reining, and Dr. F. Sottile.

${ }^{1}$ P. Hohenberg and W. Kohn, Phys. Rev. B 136, B864 (1964).

${ }^{2}$ W. Kohn and L. J. Sham, Phys. Rev. 140, A1133 (1965).

${ }^{3}$ J. P. Perdew, R. G. Parr, M. Levy, and J. L. Balduz, Phys. Rev. Lett. 49, 1691 (1982).

${ }^{4}$ J. P. Perdew and M. Levy, Phys. Rev. Lett. 51, 1884 (1983).

${ }^{5}$ L. J. Sham and M. Schlüter, Phys. Rev. Lett. 51, 1888 (1983).

${ }^{6}$ L. J. Sham and M. Schlüter, Phys. Rev. B 32, 3883 (1985).

${ }^{7}$ We work within the ensemble approach that extends the domain of the definition of density functional to fractional number of particles.

${ }^{8}$ A. Dreuw, J. L. Weisman, and M. Head-Gordon, J. Chem. Phys. 119, 2943 (2003).

${ }^{9}$ A. Dreuw and M. Head-Gordon, J. Am. Chem. Soc. 126, 4007 (2004).

${ }^{10}$ O. Gritsenko and E. J. Baerends, J. Chem. Phys. 121, 655 (2004).

${ }^{11}$ B. Champagne, E. A. Perpete, S. J. A. van Gisbergen, E. J. Baerends, J. G. Snijders, C. Soubra Ghaoui, K. A. Robins, and B. Kirtman, J. Chem. Phys. 109, 10489 (1998).

${ }^{12}$ S. J. A. van Gisbergen, P. R. T. Schipper, O. V. Gritsenko, E. J. Baerends, J. G. Snijders, B. Champagne, and B. Kirtman, Phys. Rev. Lett. 83, 694 (1999).

${ }^{13}$ W. G. Aulbur, L. Jonsson, and J. W. Wilkins, Solid State Phys., Adv. Res. Appl. 54, 1 (2000).

${ }^{14}$ G. Onida, L. Reining, and A. Rubio, Rev. Mod. Phys. 74, 601 (2002).

${ }^{15}$ R. W. Godby, M. Schlüter, and L. J. Sham, Phys. Rev. Lett. 56, 2415 (1986).

${ }^{16}$ O. Gunnarsson and K. Schonhammer, Phys. Rev. Lett. 56, 1968 (1986).

${ }^{17}$ R. W. Godby, M. Schlüter, and L. J. Sham, Phys. Rev. B 36, 6497 (1987).

${ }^{18}$ K. Schonhammer and O. Gunnarsson, J. Phys. C 20, 3675 (1987).

${ }^{19}$ O. V. Gritsenko, R. van Leeuwen, and E. J. Baerends, Phys. Rev. A 52 , 1870 (1995)

${ }^{20}$ C. Filippi, X. Gonze, and C. Umrigar, in Recent Developments and Applications of Density Functional Theory, edited by J. Seminario (Elsevier, Amsterdam, 1996).

${ }^{21}$ W. Knorr and R. W. Godby, Phys. Rev. Lett. 68, 639 (1992).

${ }^{22}$ J. D. Talman and W. F. Shadwick, Phys. Rev. A 14, 36 (1976).

${ }^{23}$ M. Stadele, J. A. Majewski, P. Vogl, and A. Görling, Phys. Rev. Lett. 79, 2089 (1997)

${ }^{24}$ M. Stadele, M. Moukara, J. A. Majewski, P. Vogl, and A. Görling, Phys. Rev. B 59, 10031 (1999).

${ }^{25}$ R. J. Magyar, A. Fleszar, and E. K. U. Gross, Phys. Rev. B 69, 045111 (2004).

${ }^{26}$ M. S. Hybertsen and S. G. Louie, Phys. Rev. B 34, 5390 (1986).

${ }^{27}$ L. Hedin, Phys. Rev. 139, A796 (1965).

${ }^{28}$ L. Hedin and S. Lundquist, Solid State Physics, (Academic, New York, 1969), Vol. 23
${ }^{29}$ U. von Barth, N. E. Dahlen, R. van Leeuwen, and G. Stefanucci, Phys. Rev. B 72, 235109 (2005).

${ }^{30}$ G. Baym and L. Kadanoff, Phys. Rev. 124, 287 (1961).

${ }^{31}$ L. Luttinger and J. Ward, Phys. Rev. 118, 1417 (1960).

${ }^{32}$ A. Klein, Phys. Rev. 121, 950 (1961).

${ }^{33}$ An infinite set of functionals can be constructured by adding a functional $F[D]$ where $D[G]=G\left(1 / G_{s}-\Sigma_{\text {xc }}\right)-1$, such that it obeys $F[0]$ $=d F[0] / d D=0$ when the $G$ satisfies Eq. (2.2). See Ref. 29 and references therein.

${ }^{34} \mathrm{~A}$ different strategy is adopted instead in the hydrids/generalized KS schemes (Refs. 68-70) that use a nonlocal (static) potential corresponding to the orbital dependent energy expression.

${ }^{35}$ Y. M. Niquet, M. Fuchs, and X. Gonze, Phys. Rev. A 68, 032507 (2003).

${ }^{36}$ A. Görling, Phys. Rev. B 53, 7024 (1996).

${ }^{37}$ C. O. Almbladh, U. Von Barth, and R. Van Leeuwen, Int. J. Mod. Phys. B 13, 535 (1999).

${ }^{38}$ Y. M. Niquet and X. Gonze, Phys. Rev. B 70, 245155 (2004).

${ }^{39}$ A. Marini, G. Onida, and R. Del Sole, Phys. Rev. Lett. 88, 016403 (2002).

${ }^{40}$ The parameters $\widetilde{\omega}$ and $R$ are determined by forcing the model to reproduce the dielectric matrix in the zero and plasmon-pole frequencies. See Ref. 17.

${ }^{41}$ J. B. Krieger, Y. Li, and G. J. Iafrate, Phys. Rev. A 46, 5453 (1992).

${ }^{42}$ M. E. Casida, Phys. Rev. A 51, 2005 (1995).

${ }^{43}$ O. V. Gritsenko and E. J. Baerends, Phys. Rev. A 64, 042506 (2001).

${ }^{44}$ M. Grüning, O. V. Gritsenko, and E. J. Baerends, J. Chem. Phys. 116, 6435 (2002)

${ }^{45}$ X. Gonze, J. M. Beuken, R. Caracas, et al., Comput. Mater. Sci. 25, 478 (2002).

${ }^{46}$ N. Troullier and J. L. Martins, Phys. Rev. B 43, 1993 (1991).

${ }^{47}$ M. Fuchs and M. Scheffler, Comput. Phys. Commun. 119, 67 (1999).

${ }^{48}$ D. M. Ceperley and B. J. Alder, Phys. Rev. Lett. 45, 566 (1980).

${ }^{49}$ J. P. Perdew and A. Zunger, Phys. Rev. B 23, 5048 (1981).

${ }^{50}$ H. Monkhorst and J. Pack, Phys. Rev. B 13, 5188 (1976).

${ }^{51}$ Computer code SELF http://www.fisica.uniroma2.it/ self/.

${ }^{52}$ E. Engel, A. Hock, R. N. Schmid, R. M. Dreizler, and N. Chetty, Phys. Rev. B 64, 125111 (2001).

${ }^{53}$ Semiconductors: Physics of Group IV Elements and III-V Compounds, Landolt-Börnstein, New Series, Group III, Vol. 17 Pt. A, edited by O. Madelung, M. Schulz, and H. We (Springer, Berlin, 1982).

${ }^{54}$ J. Ortega and F. Himpsel, Phys. Rev. B 47, 2130 (1993).

${ }^{55}$ M. Piacentini, G. Lynch, and C. Olson, Phys. Rev. B 13, 5530 (1976).

${ }^{56}$ R. Haensel, G. Keitel, M. Koch, E. E. Skibowski, and P. Schreiber, Phys. Rev. Lett. 23, 1160 (1969).

${ }^{57}$ J. Gräfenstein, E. Kraka, and D. Cremer, J. Chem. Phys. 120, 524 (2004).

${ }^{58}$ E. J. Baerends and O. Gritsenko, J. Chem. Phys. 123, 062202 (2005).

${ }^{59}$ Topics in Condensed Matter Physics (Nova Science, New York, 1994), Chap. 7, p. 121.

${ }^{60}$ T. Kotani, J. Phys.: Condens. Matter 10, 9241 (1998).

${ }^{61}$ For molecules the difference between the Klein and Luttinger-Ward functional has been studied in Ref. 71.

${ }^{62}$ X. Gonze and M. Scheffler, Phys. Rev. Lett. 82, 4416 (1999).

${ }^{63}$ I. V. Tokatly and O. Pankratov, Phys. Rev. Lett. 86, 2078 (2001).

${ }^{64}$ L. Reining, V. Olevano, A. Rubio, and G. Onida, Phys. Rev. Lett. 88, 066404 (2002).

${ }^{65}$ A. Marini, R. Del Sole, and A. Rubio, Phys. Rev. Lett. 91, 256402 (2003).

${ }^{66}$ F. Bruneval, F. Sottile, V. Olevano, R. Del Sole, and L. Reining, Phys. Rev. Lett. 94, 186402 (2005).

${ }^{67}$ Y. H. Kim and A. Görling, Phys. Rev. Lett. 89, 096402 (2002).

${ }^{68}$ A. D. Becke, J. Chem. Phys. 98, 5648 (1993).

${ }^{69}$ A. Seidl, A. Görling, P. Vogl, J. A. Majewski, and M. Levy, Phys. Rev. B 53, 3764 (1996).

${ }^{70}$ A. Görling and M. Levy, J. Chem. Phys. 106, 2675 (1997).

${ }^{71}$ N. E. Dahlen, R. Van Leeuwen, and U. Von Barth, Int. J. Quantum Chem. 101, 512 (2005). 